En su conferencia Was soll und kann Literatur verändern? Erich Fried reflexionaba sobre sus lecturas y vivencias, y cómo afectaban a su forma de escribir. Los traductores, como escritores que son, también desarrollan una poética propia. Los procesos creativos de ambos se ven guiados y limitados por la capacidad de crear verosimilitud, alimentada de dichas lecturas y vivencias. Hoy que la didáctica de la Traducción se centra en las capacidades prácticas, dejando de lado la cultura general, los traductores noveles tienden a carecer de herramientas para resolver la interpretación textual de forma coherente. Quizá la literatura pueda proporcionarles dichas herramientas.

PALABRAS CLAVE: Traducción, poética traductora, lectura, proceso creativo, cultura.

\title{
¿Qué puede cambiar la literatura para los traductores?
}

ItZIAR HeRnÁndeZ Rodilla

Universidad Complutense de Madrid
What could literature change for translators?

In his lecture Was soll und kann Literatur verändern?, Erich Fried spoke about his readings and experiences, what he asked from them and how they affected his writing. In the same way writers develop their poetics, translators develop theirs when translating. There is a certain parallelism in their creative process, guided and restricted by their ability to create verisimilitude, fuelled by the things they read. Today, when the didactics of Translation focus on practical abilities and technology, leaving aside general culture, recent graduates tend to lack the criteria to solve translation problems in a resolute and consistent way. Literature could give them the tools to solve such problems.

KEY WORDS: Translation, translation poetics, reading, creative process, culture. 
Esta nota comenzará con una excusatio: lo que se expone a continuación es menos el resumen de una investigación que el relato de una reflexión. Y esta no es siquiera original, como será posible deducir de algunas de las fuentes citadas, que se remontan a los poetas romanos o al Siglo de Oro español. Es un asunto este que me inquieta personalmente desde hace algún tiempo, que se abrió paso hasta las conclusiones de la tesis que presenté a comienzos de 2016, titulada ... und alle seine Mörder... Erich Fried traducido para el teatro, y que se hizo más profundo como inquietud cuando comencé a dar clases de Traducción en la universidad en el segundo semestre del curso 2015-2016. Como he mencionado, el planteamiento no es original y esta nota ni siquiera tiene la intención de proponer una solución. Se presenta, de hecho, como invitación a la reflexión y el debate, y casi para encontrar, con ello, la excusa, esta vez como pretexto, que pueda provocar una consideración más seria del tema, y ello empezando en el mismo lugar que la preocupación original, por la poética de Erich Fried.

Viajemos, pues, de vuelta a la Universidad de Viena en I983; al simposio Literatur und Macht [Literatura y poder], donde Erich Fried, iniciando el tema del poder de la literatura, de lo que esta debe y puede cambiar, se dirige a su público hablando de otro poeta, Hugo Sonnenschein, Sonka: «Sonkas Schreiben [hat] sich zwar unter dem Eindruck seiner Erfahrung verändert, $[\ldots]$ er [ist] sich aber treu geblieben ${ }^{\mathrm{I}}$ » (Fried, r983: 76).

Reconoce así, en cierta manera, la existencia y la evolución de la poética de un autor y la lealtad que dicho autor le debe: la literatura puede no

I La escritura de Sonka ha cambiado, a decir verdad, por efecto de sus experiencias, pero él se ha mantenido fiel a ella. [La traducción (como todas las demás que aparecerán en la nota, salvo indicación contraria) es de la autora]. ser leal a sí misma, dice Fried, pero el autor debe saber mantener su fidelidad.

La literatura cambia en sí misma, pues, y también al que la escribe y al que la lee o la escucha, según afirma Fried (I983: 77): «Was soll und kann Literatur verändern? Ich glaube, zuerst einmal den Schreibenden selbst und zweitens den Leser oder den Hörer».

Al que escribe porque, en la necesidad de formular sus pensamientos, aclara sus opiniones en cuanto a diversos asuntos; porque, además, la escritura es también un proceso de conocimiento y el conocimiento no tiene, por lo general, vuelta atrás. Con Ibsen, opina que escribir tiene algo de autocrítica y, con Goethe, que permite expresar de forma legítima el sufrimiento y la protesta. Recurriendo a las poéticas de otros, avanza inexorable hacia una definición de la suya propia - en I983 tiene 62 años y ya le han operado por primera vez del cáncer que acabará con su vida cinco años más tarde-, que resume en un «intento de supervivencia»: "Tatsächlich waren und sind die besten Dichter im allgemeinen die, die nicht dichten konnten, sondern die dichten mußten, um überhaupt dieses Leben $\mathrm{zu}$ ertragen, oder es wenigstens möglichst lange $\mathrm{zu}$ ertragen $^{2} »$ (Fried, I983: 77).

$\mathrm{Y}$, por eso, la actualidad de un escritor se refleja en su escritura no solo si se dedica a la agitación o la propaganda. La tarea del arte, de la literatura es, en última instancia, comprometer al hombre con su vida, evitar que se habitúe, que se embote, que se enajene de la sociedad, que deje de verse como protagonista de su entorno, se sienta ajeno a él, se aísle y, como consecuencia, se pierda de sí mismo:

2 En realidad, los mejores escritores eran y son, por lo general, no quienes «pueden» escribir, sino quienes «tienen que» hacerlo, en resumidas cuentas, para aguantar la vida o para aguantarla, por lo menos, a ser posible, durante largo tiempo. 
[Die Literatur] Wofür aber engagiert? Nicht für eine Partei $[\ldots]$ sondern engagiert gegen die Entfremdung, gegen das Nicht-mehrsehen, Nicht-mehr-fühlen, Nicht-mehr-merken-wollen, was mit uns geschieht und was wir einander tun. Und das glaube ich — gegen das Stumpfwerden, gegen das Gedankenloswerden zu schreiben - ist eigentlich das, was Literatur soll und auch kann ${ }^{3}$ (Fried, I983: 78).

Este es el objetivo principal, la razón de ser, del trabajo de un hombre que afirmaría en uno de sus poemas: «Ich will mich erinnern / an alles was man vergißt4» (Fried, 2007 [1995]: I2I). Con ello cumple uno de los principales mandamientos de la cultura hebrea de la que procede: Zachor! — ¡Recuerda!- , que aparece en la Biblia más de ciento setenta veces y que los judíos siguen hoy más que ningún otro pueblo (Schäfer, I998: 85). Y, a través de ese «no olvidar», de esa «lucha contra la enajenación ${ }^{5}$ — Contra la «alienación» diría la teoría marxista-, pretende cambiar un mundo con el que nunca acabará de estar satisfecho, pues eso supondría haber dejado de sentir o haber conseguido su objetivo.

Asimismo, en la búsqueda de la formulación exacta de sus pensamientos, el escritor hará que

3 (La literatura) ¿Comprometida con qué? No con un partido (...) sino contra la enajenación, contra el dejar de ver, el dejar de sentir, el dejar de querer darse cuenta de lo que nos sucede y de lo que nos hacemos unos a otros. $Y$ eso, creo - escribir contra el embotamiento, contra la irreflexión - es, en realidad, lo que la literatura debe y puede hacer.

4 Versos del poema «Gegen Vergessen» (Contra el olvido): Quiero acordarme de / todo lo que se olvida.

5 Erich Fried atribuye el origen de su principio poético, la «lucha contra la enajenación», al Che Guevara, que aclaraba en su carta El socialismo y el hombre en Cuba: «E1 hombre, en el socialismo, a pesar de su aparente estandarización, es más completo. [...] Todavía es preciso acentuar su participación consciente, individual y colectiva, en todos los mecanismos de dirección y de producción [...]. Así logrará la total consciencia de su ser social, lo que equivale a su realización plena como criatura humana, rotas todas las cadenas de la enajenación» (Guevara, 1965). sucedan cosas con las palabras: «Eine Dichtung, die nur inhaltlich Interessantes bringt, wo mit der Sprache selbst überhaupt nichts geschieht, ist selten sehr gute Dichtung ${ }^{6}{ }$ (Fried, I983: 79).

Y continúa hablando de la escritura de Christa Wolf, de política contemporánea, de Goethe una vez más, de Marx a continuación; de Sylvia Plath, del consuelo que encuentra en Hölderlin y en Kafka, y de lo que le enseñó Paul Celan; dando con ello pistas sobre su propia literatura - comprometida siempre-y sus motivaciones para escribir:

Literatur kann Menschen verändern und kann die Welt verändern, nicht direkt, sondern dadurch, daß sie das Denken und Fühlen einzelner Menschen wach erhält. Sie hat auch therapeutische Wirkungen, dadurch, daß sie Menschen daran hindern kann, an Problemen, die unbewußt bleiben, zusammenbrechen. Auch das ist ein wichtiger Einfluß zur Veränderung der Wirklichkeit ${ }^{7}$ (Fried, I983: 8I).

Para acabar diciendo: «Wir müssen die Klischees der Zivilisation, die zum Schutz der Unmenschlichkeit dienen, durchbrechen, wo immer wir können" ${ }^{8}$ (Fried, I983: 85).

Es patente, pues, que la obra de Erich Fried bebe de una riqueza inconmensurable de fuentes: su origen judío y vienés, que se combinaría en el exilio con los autores de habla inglesa y alemanes, por considerar el alemán su verda-

6 Una obra que solo sea interesante por su «contenido", en la que no pasa nada con el idioma, no suele ser una buena obra.

7 La literatura puede cambiar a los hombres y el mundo, no directamente, sino en la medida en que mantiene despierto el pensamiento y los sentimientos de cada hombre. Tiene, asimismo, efectos terapéuticos, de tal manera que puede evitar que los hombres se desmoronen por problemas que no se conocen. También eso es una importante influencia para cambiar la realidad.

8 Es nuestro deber romper los clichés de la civilización que protegen la barbarie siempre que podamos.

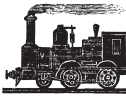


dera patria e Inglaterra su patria adoptiva; la situación sociopolítica contemporánea, de gran complejidad en la época que le tocó vivir, y su intención de trabajar el lenguaje como medio de dar forma al mundo.

Pasando a la tarea del traductor, se propone como punto de partida el resumen de las posturas clásicas que lleva a cabo Amparo Hurtado (2008 [200I]: 3II, 367) y la necesidad propuesta por dichos modelos de considerar el análisis de los textos de modo integral como paso primero e inexcusable que permita la interpretación y la captación del sentido para asegurar la correcta comprensión e interpretación del sentido del texto, y para establecer los criterios sobre los que asentar las decisiones en cuanto a las estrategias y procedimientos que adoptar para la traducción. Serán precisos para la correcta interpretación del texto el análisis, asimismo, de las convenciones estilísticas del contexto en que se escribió, así como su relación con el entorno y el momento culturales, políticos, religiosos e ideológicos que, desde el punto de vista comunicativo, aportarán información sobre las intenciones del autor y los conocimientos que comparte con su público original. Por otra parte, desde un punto de vista pragmático, será el estudio de las intenciones del autor y sus formas, su estilo, lo que podrá sugerir pistas en cuanto a los sistemas de creencias, aspectos culturales, ideología, percepciones y actitudes que entraña el original y que se deberán transmitir al público del texto traducido. Este aspecto se verá indudablemente complementado con aspectos semánticos extratextuales relacionados con la selección léxica que realiza el autor: el tiempo y el lugar en que escribe, quién es, cuáles son sus intenciones, quién o quiénes son sus receptores, el medio, la función del texto o el significado con que hace uso de determinados vocablos.
En el caso de Erich Fried, serán pues destacables:

- su compromiso político antibélico y antifascista, marcado por su «lucha contra la enajenación» — tan relacionada con la educación tras Auschwitz promulgada por Adorno (1977) - y los recurrentes temas de la reconciliación y la culpa, incluida la del superviviente, que aparecen una y otra vez en su obra lírica;

- su calidad de puente entre dos culturas: la abandonada en Austria, pero mantenida en su «patria intelectual» como escritor en lengua alemana, y la de acogida en Inglaterra;

- su capacidad de reacción ante los acontecimientos que le fueron contemporáneos, tanto a nivel local como a nivel mundial, entre los que se encuentran, por ejemplo, la reintegración a la vida política alemana de exnazis o la guerra de Vietnam, o la política antiterrorista alemana;

- y su estilo, que jugaba con la lengua alemana utilizando las reglas de la inglesa, gracias a su vida en y entre los dos idiomas.

El traductor de Fried debe, pues, consciente de la intertextualidad, generar una poética propia paralela, según la que llevar a buen término el proceso traductor. Una poética que le permita, como decía Hemingway — referencia sin igual a la hora de definir el resbaladizo concepto de la cultura humanística- contar con un «detector de basura» interior para descartar propuestas inverosímiles y aprovechar las que dotan de verosimilitud a la escritura, en este caso, a la traducción de un autor. Aunque es una idea antigua. Ya se refería a ella Persio Flaco — que murió en el 62 d. C.- en su primera sátira contra los escritores que se convertían repentinamente en poetas: el saber es el comienzo y la fuente de la escritura correcta, a aquel que 
conoce bien las cosas se le ocurren las palabras; y la poesía, por lo tanto, no solo exige talento y el conocimiento de las reglas para poetizar, sino también el saber sobre la realidad misma (Strosetzki, 1997: 2I4-215).

Pero de vuelta a la traducción que, como decía Octavio Paz (1971: r64), es operación gemela de la creación poética, para conservar la intertextualidad, el traductor debería andar casi en paralelo con el autor para reflejar las influencias de este en la traducción. Tampoco es nueva la idea. Sánchez de las Brozas - profesor del Colegio Trilingüe de Salamanca-, en su obra De auctoribus interpretandis, sive de exercitatione (I58r: 3), decía que quien desee comprender un texto ajeno está obligado a disponer él mismo de las reglas y el saber que utilizó el autor y que, para disminuir la distancia entre autor e intérprete, se pueden poner en mano de este las reglas que siguieron otros autores para la elaboración de sus escritos. Es una primera indicación de cómo usar la literatura de la misma forma que lo hace un autor para crear su poética. Más adelante, Carrillo y Sotomayor, en su Libro de la erudición poética (I61r: 55), ${ }^{9}$ consciente de que el habla del poeta es oscura para la mayoría del pueblo, explica que la redacción de una obra literaria es un intensivo trabajo que dispone incluso de los conocimientos de los filósofos, y que el intérprete tiene, por tanto, que disponer de una amplitud de conocimientos que se corresponda con los que tiene el autor. Así, el que se proponga interpretar a un poeta, no solo debe tener conocimientos de las diferentes corrientes filosóficas, sino también de derecho, medicina, dialéctica e incluso de todas las disciplinas y disponer de un saber enciclopédico. Luis Vives (De causis corruptarum artium, I53I;

\footnotetext{
9 Se cita a ambos, Sánchez de las Brozas y Carrillo y Sotomayor, según Christoph Strosetzki (1997: 212-216).
}

en Opera omnia, t. 6, pp. 44 y ss.) abunda, en este sentido, en la necesidad de que el intérprete, como opuesto al gramático que se ocupa de los principios lingüísticos, sea un humanista que puede, por una parte, investigar sobre la Antigüedad, de tal forma que consiga formarse una imagen lo más objetiva posible de ella, y por otra, interesarse, sobre todo, por las cuestiones de su propio presente y solamente buscar en la Antigüedad las soluciones a los problemas, utilizando la literatura clásica como colección de materiales modélicos para sus debates orientados hacia el presente, que resolverá imitando los ejemplos de la literatura y el pensamiento clásicos (Strosetzki, 1997: 343-352).

Más cerca de la actualidad, Adam Kovacsics (20II) cita a Osip Mandelstam. Humanista convencido defensor de la cultura, esta vez en la Rusia de la revolución soviética, Mandelstam se pronunciaba en 1922 decididamente contra la concepción utilitaria del lenguaje, que podía acabar en pura manipulación, a favor de una cultura que no empieza de cero, sino que parte de Mozart, Schubert o Shakespeare. A su juicio, lejos de ser personajes ajenos al ser humano, estos creadores expresaban formas e inquietudes universales y no se hallaban por encima del pueblo ni al margen de la vida real.

Se puede percibir, en toda esta evolución de autoridades, la misma idea. El intérprete, el traductor, debe saberlo, de alguna forma, todo. Principalmente, desde luego, lo que se refiere al autor del texto que le ocupa. Lo que implicaría que su formación fuese, eminentemente, humanista clásica.

Ahora bien. Hoy, cuando es materia de discusión si una persona culta no debería saber también cómo funciona un ordenador, entender la trascendencia del bosón de Higgs o conocer los principios de la mecánica cuántica, tendemos a centrar la formación de los traductores

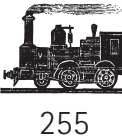

255 
en «el idioma que se forja y manipula en otras [disciplinas] más populares y abundantes». La cita es de Xosé Castro (1999) refiriéndose, precisamente, al lenguaje informático y de los manuales técnicos que constituyen cada año un porcentaje de lectura mucho mayor que la literatura. Se enseña a los futuros traductores a manejar programas informáticos que es posible que no lleguen a ver nunca en su vida profesional y a saber cómo traducir novela romántica o tacos, descendiendo la formación literaria al nivel que tienen, sin retarles a avanzar, a llegar allí donde encontrarían el saber de los autores.

Se tropieza hoy, además, con una generación —la de los llamados «millenials» - convencida de que, el ser la más formada —en extensión democrática y oportunidades - e informada - puesto que, en sus palabras, tienen «todo el conocimiento del mundo [...] en su bolsillo, a un solo clic de distancia» (Tatay, 20I6)—; y al estar educada en una sociedad en la que, ya desde el Siglo de Oro, el trabajo intelectual, la vita contemplativa, pierde valor frente al capitalismo y la concepción positiva del trabajo burgués con una utilidad como factor de la producción sobre el que se asienta la economía (Strosetzki, I997: I), no ve un problema, sino una ventaja, en que su capacidad de atención se haya reducido a un segundo por debajo de la del paradigmático pez común. Lejos de instarles a la contemplación, este hecho les impulsa a hallar la respuesta «sin leer el manual de instrucciones, que es de cobardes» (Tatay, 20r6). Algo que está muy bien si integra el fracaso como forma del proceso de aprendizaje, pero que, en la saturación de información a la que vivimos sometidos, atasca el pensamiento crítico para poder sobrevivir y deja de alimentar el «detector de basura» que el profesional va a necesitar. Un pensamiento crítico que es, además, determinante para los glorificados con- textos de creatividad y pensamiento divergente. Como han averiguado los neurobiólogos, este pensamiento depende de lo que se llama «actos bisociativos». Esa lucecita que se nos enciende fulgurante en forma de idea, metáfora de la invención, se debe a la capacidad de nuestra mente de unir dos piezas de información que se han memorizado en ella. Es decir, la invención depende de la existencia de información en nuestra memoria. Y, si esa información previa no existe, será imposible inventar.

Supone, desde luego, un reto formar a una generación que necesita misiones épicas, dignas de videojuego, pero que no tiene la paciencia de viajar a Ítaca para adquirir la información que le permita llegar a destino.

Como se ha mencionado al principio, esta nota está lejos de poder proponer una solución. Aunque la opinión de quien la escribe es que dicha solución pasa por la literatura.

La cultura es algo complejo: un ideal, un proceso, un conjunto de conocimientos y de capacidades, y un estado. Pero es, además y entre otras cosas, un juego — también social-, en el que los jugadores han olvidado la mayor parte de sus conocimientos, pero en el que los conocimientos que aún conservan les recuerdan los que les faltan. Es decir, saben lo que una vez supieron y conocen las reglas del juego. Así que siempre son capaces de jugar igual de bien, aunque sea de forma reactiva y no proactiva.

La cultura de un individuo no se compone fundamentalmente de datos, sino más bien, como en el caso del jugador de ajedrez, de una mezcla de reglas, informaciones y de una visión global del terreno de juego, así como del número de piezas y de su valor. Si tiene esto, podrá recordar lo que ha perdido y conservará intacta la capacidad de jugar, aunque haya olvidado muchos de sus conocimientos. Al igual que 
Sócrates, sabe perfectamente lo que no sabe (Schwantz, 2005: 69I-718).

Quizá esa sería una buena forma de vender la cultura a futuras generaciones: como un juego de apariencias, en el que, más que nunca, el ser humano en sociedad está envuelto.

En cualquier caso, volviendo al tema, la literatura se presenta como el medio de aprender esas reglas. Como decía Fried, el arte, la literatura, comprometen al hombre con su vida, pero también le ofrecen la posibilidad de formular a manera de cita las complejas relaciones existentes entre determinados procesos sociales y las vidas de las personas, dándoles un nombre y una dirección. En sus historias se personifican destinos prototípicos, que después adquieren una fisonomía concreta a través de los correspondientes personajes: Hamlet, Don Juan, Fausto, Lady Macbeth, Ana Karénina, etcétera. Al igual que las personas, estos personajes son «informaciones condensadas».

Asimismo, el tiempo solo puede observarse a través de las historias, pues solo ellas of recen la lógica de determinados procesos. Y, solo a través de las historias que observamos en otros, podemos observar los procesos en los que nos hallamos inmersos nosotros mismos. Como dice Schwantz, si no se conoce a Don Quijote, resulta más fácil enredarse en luchas contra molinos de viento; si no se ha leído Las brujas de Salem de Arthur Miller es más probable llegar a formar parte de una jauría inconsciente que va a la caza de una presa. Únicamente a través de las obras de la literatura podemos tomar distancia con respecto a nosotros mismos. Porque hay algo que solo la novela o la poesía pueden ofrecer en las historias que, ahora, llegan masivamente a través del cine y la televisión: la visión interna de un personaje. Solo la novela nos permite experimentar cómo puede sentirse la víctima de una jauría humana.
Es, además, en la literatura, como también nos recuerda Fried, donde se reflejan las invenciones, el entorno político, cultural, social, incluso económico (piénsese, por ejemplo, en el paradigma de burbuja financiera que ofrece El tulipán negro de Dumas padre), que ha experimentado fictivamente o vivido el autor, dándonos con ello y sin necesidad de investigaciones profundas, información experta sobre diversos temas.

$\mathrm{O}$, en términos más traductológicos, los conocimientos - lingüístico, claro, pero también metalingüístico, textual, contextual y del mundo- que necesitaremos como traductores para entretejer los vacíos entre ellos, como decía Nicholas Round (2005), y conseguir con ello una red que recoja las posibilidades del idioma en el texto de partida en su traslado al idioma de llegada.

Decía, por último, Enrique Bernárdez (2000) que es imposible que un traductor sepa todo lo que va a tener que saber alguna vez y que lo mejor es que esté preparado para cualquier imprevisto. Descubrir al traductor principiante la cultura humanística es, en ese sentido, la mejor herramienta que podemos dar a quienes aspiran a contarse entre los profesionales.

También como experiencia vital, si vamos a ello y volvemos a Fried. La literatura puede salvar la vida a quien escribe. Pero también al que la lee, evitando que se aliene. Dándole herramientas de conocimiento externo y propio. Porque, como decía Saavedra Fajardo en su República literaria (1655), no debe considerarse sabio al que no sabe nada de sí mismo.

RECIBIDA EN DICIEMBRE DE $20 I 7$ ACEPTADA EN DICIEMBRE DE $20 I 7$ VERSIÓN FINAL DE SEPTIEMBRE DE $20 I 8$

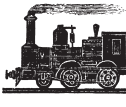




\section{BIBLIOGRAFÍA}

Adorno, Theodor W. (1967): «La educación después de Auschwitz», conferencia originalmente realizada por la Radio de Hesse, el i8 de abril de I966; publicada, posteriormente, en Theodor W. Adorno Zum Bildungsbegriff des Gegenwart, Fráncfort del Meno: Diesterweg, iII y ss.

- (1977): Gesammelte Schriften Band ro: Kulturkritik und Gesellschaft I/II, Fráncfort del Meno: Suhrkamp Verlag.

Bernárdez, Enrique (2000): "Traducción y cultura general», El Trujamán del Centro Virtual Cervantes, I6 de mayo, <http://cvc.cervantes.es/ trujaman/anteriores/mayo_oo/I605200o.htm > [consulta: I3.XII.2OI7].

Castro, Xosé (I999): "La cultura traducida», El Trujamán del Centro Virtual Cervantes, i8 de noviembre, <http://cvc.cervantes.es/trujaman/ anteriores/noviembre_99/r8III999.htm > [consulta: I3.XII.2OI7].

Fried, Erich (I983): «Was soll und kann Literatur verändern?», Wespennest, 52, 76-83.

- (2007): Gedichte, Múnich: DTV, r. ${ }^{\mathrm{a}}$ ed.: 1995.

Guevara, Ernesto (1965): «El socialismo y el hombre en Cuba», carta publicada por Carlos Quijano en el semanario uruguayo Marcha, I2 de marzo.

Hurtado Albir, Amparo (2008): Traducción y Traductología. Introducción a la Traductología, Madrid: Cátedra, I. ${ }^{\text {a }}$ ed.: 200 I.

Kovacsics, Adam (20II): "Osip Mandelstam: palabra, cultura, traducción», El Trujamán del
Centro Virtual Cervantes, I8 de agosto, <http:// cvc.cervantes.es/trujaman/anteriores/agosto_II/I80820II.htm> [consulta: I3.XII.20I7].

PAz, Octavio (I97I): Traducción: literatura y literalidad, Barcelona: Tusquets.

Round, Nicholas (2005): «Translation and its Metaphors: The $(N+\mathrm{I})$ Wise Men and the Elephant», SKASE Journal of Translation and Interpretation, I (I, 2005), 47-69.

Saavedra Fajardo, Diego de (2008): República literaria, ed. de Fco. Javier Díez de Revenga, Murcia: Real Academia Alfonso X el Sabio, <www. cervantesvirtual.com/descargaPdf/republicaliteraria--3/> [consulta: I3.XII.2OI7].

SchäFer, Katrin (I998): «Die andere Seite»: Erich Frieds Prosawerk. Motive und Motivationen seines Schreibens, Viena: Ed. Praesens.

Schwantz, Dietrich (2005): La cultura. Todo lo que hay que saber, trad. de Vicente Gómez Ibáñez, Madrid: Suma de Letras.

Strosetzki, Christoph (I997): La literatura como profesión. En torno a la autoconcepción de la existencia erudita literaria en el Siglo de Oro español, Kassel: Reichenberger.

TAtay, María (20I6): «Trabajadores y millennials», vídeo de la intervención de María Tatay en la I3. ${ }^{a}$ edición del seminario de charlas ultrarrápidas Ignite Valencia, celebrado en Valencia el 30 de junio de 20I6, publicado el I8 de julio, <https:// www.you tube.com/watch?v=bvHTzhXIZUA >, [consulta: I3.XII.2OI7]. 